\title{
Mental Health First Aid guidelines for helping a suicidal person: a Delphi consensus study in the Philippines
}

\author{
Erminia Colucci ${ }^{1 *}$, Claire M Kelly², Harry Minas ${ }^{1}$, Anthony F Jorm², Dinah Nadera ${ }^{3}$
}

\begin{abstract}
Background: This study aimed to develop guidelines for how a member of the Filipino public should provide mental health first aid to a person who is suicidal.

Methods: The guidelines were produced by developing a questionnaire containing possible first aid actions and asking an expert panel of 34 Filipino mental health clinicians to rate whether each action should be included in the guidelines. The content of the questionnaire was based on a systematic search of the relevant evidence and claims made by authors of consumer and carer guides and websites. The panel members were asked to complete the questionnaire by web survey. Three rounds of the rating were carried and, at the end of each round, items that reached the consensus criterion were selected for inclusion in the guidelines. During the first round, panel members were also asked to suggest any additional actions that were not covered in the original questionnaire (to include items that are relevant to local cultural circumstances, values, and social norms). Responses to these openended questions were used to generate new items.
\end{abstract}

Results: The output from the Delphi process was a set of agreed upon action statements. The Delphi process started with 138 statements, 48 new items were written based on suggestions from panel members and, of these 186 items, 102 met the consensus criterion. These statements were used to develop the guidelines appended to this paper. The guidelines are currently being translated into local languages.

Conclusions: There are a number of actions that are considered to be useful for members of the public when they encounter someone who is experiencing suicidal thoughts or engaging in suicidal behaviour. Although the guidelines are designed for members of the public, they may also be helpful to non-mental health professionals working in health and welfare settings.

\section{Background}

As reported by WHO [1], suicide is a huge but largely preventable public health problem, causing almost half of all violent deaths and resulting in one million fatalities every year (almost 3000 every day) worldwide, as well as economic costs in the billions of dollars. Estimates suggest fatalities could rise to 1.5 million by 2020 [2]. For every person who completes suicide, 20 or more make a suicide attempt, resulting in injury, hospitalization, and emotional and mental trauma, although no reliable data are available on its full extent $[1,2]$.

\footnotetext{
* Correspondence: ecolucci@unimelb.edu.au

'Centre for International Mental Health, Melbourne School of Population Health, The University of Melbourne, Parkville, Victoria 3010, Australia Full list of author information is available at the end of the article
}

Worldwide, suicide rates have increased by $60 \%$ over the last 50 years. The increase has been especially marked in developing countries [1]. The increase has been particularly alarming amongst young people aged 15 to 25 years [2]. Although suicide rates in the Philippines are relatively low, the number of suicide-related deaths has been increasing in the past decade and most of suicides are in the 15-to-24 and 75-and-above age groups [3].

For every suicide death there are scores of family and friends whose lives are devastated emotionally, socially and economically [2]. For family members and friends affected by suicide or attempted suicide the emotional impact may last for many years [1].

A member of the community who is close to the suicidal person, such as a friend, family member, co-worker 
or classmate, is likely to be the first person to notice suicide warning signs. However, few have the knowledge and skills required to recognize the imminent risk of suicide and to assist in preventing suicide. Simple and practical guidelines on how a member of the public should provide first aid to a person who is suicidal (i.e. has expressed suicidal thoughts or intent, whether overt or covert, or has taken action toward making a suicide attempt) might help such a person to encourage a suicidal individual to seek professional help or decide against suicide. Such guidelines can be applied in training courses for the public.

First aid training is widespread throughout the world, giving members of the public skills to help an injured person before medical help arrives. There are many organizations offering first aid training, but the first aid practices taught in these courses generally conform to national guidelines. While first aid training is common, it generally ignores mental health crises such as how to assist a suicidal person. Nevertheless, there have been efforts to develop training for the public that does cover these issues, such as Applied Suicide Intervention Skills Training (ASIST) [4] and Mental Health First Aid (MHFA) training [5]. Unfortunately, there is limited evidence to guide the content of such training. While randomized controlled trials provide the highest standard of evidence, it is not feasible or ethical to carry out such trials to evaluate specific suicide first aid strategies. In the absence of high quality evidence, the best option for developing guidelines is expert consensus. There are formal methods for assessing expert consensus that have been used in several areas of health research. One of the most commonly used consensus method is the Delphi process [6]. There are many variants but all involve a group of experts making private ratings of level of agreement with a series of statements, feedback to the group of a statistical summary of the ratings, and then another round of rating. Delphi group members do not meet, so it is possible to do studies using mail or the Internet. The output from the process is statements for which there is substantial consensus in ratings. The Delphi method has been used in health research since the mid-1970s [7]. The Delphi method has been used to develop suicide first aid guidelines for developed English-speaking countries [8], as well as mental health first aid guidelines for nonsuicidal self injury [9], panic attacks [10], psychosis [11], depression [12], eating disorders [13], problem drinking [14] and traumatic events [15]. However, guidelines produced in these contexts will not necessarily be applicable in countries with different cultures and health systems. We therefore wished to explore the possibility of developing suicide first aid guidelines for a number of Asian countries. This project was undertaken to establish whether the use of the Delphi method is feasible in the development of suicide first aid consensus guidelines for Asian countries. This method was previously successfully implemented in the production of first aid guidelines for psychosis in Asia [11].

The aim of this project was to produce guidelines for use in particular Asian countries on how a member of the public should provide first aid to a person who is suicidal. The project did not aim to test hypotheses, rather to develop guidelines on first aid actions based on the consensus of expert clinicians. The project involved undertaking separate studies in three countries: Japan, Philippines and India. These three countries were chosen because they are Asian countries with different cultural and religious contexts, different rates of suicide, different levels of economic development, and different levels of availability of mental health services. We expected that there would be differences across countries in the views expressed by the expert panels about appropriate guidelines for mental health first aid in relation to suicide [16-19]. This paper presents the results of the study in the Philippines. The results from India have been published [20], whereas those from Japan will be described in a subsequent article. To the best of the authors' knowledge, no study of this kind has been conducted in these countries before.

\section{Methods}

The first aid guidelines were produced using: (a) a systematic search of the relevant evidence and claims made by authors of consumer and carer guides and websites; (b) development of a questionnaire on possible first aid actions which was based on the search; (c) and the consensus of panels of clinicians from each of the countries on which first aid actions should be included in the guidelines.

\section{Systematic search for possible suicide first aid actions in the literature}

As part of the project to develop suicide first aid guidelines for developed English-speaking countries, a systematic search for possible first aid actions was carried out in the formal professional literature listed in PubMed and PsycLit and other sources such as existing general mental health first aid manuals [5], other relevant manuals and guides on suicide prevention (e.g. Suicide Prevention Skills Training, [21]; Mental Health for Emergency Departments [22]) and relevant web sites (e.g. Samaritans). This method has been previously published for suicide first aid guidelines in developed English-speaking countries [8].

\section{Construction of the questionnaire}

A questionnaire was constructed from a content analysis of the actions indicated in the literature. Only statements 
that suggested a potential first aid action (i.e. what the first aider should do) or relevant awareness statements (what the first aider should know) were included in the questionnaire. These statements were grouped into common themes and used by a working group to generate questionnaire items specifying what actions a first aider should take. No judgments were made by the working group about the potential usefulness of the statements. Anything was included that fitted the definition of first aid, even if contradictory to other statements.

The questionnaire developed for English-speaking countries had 114 items, each describing a potential action that a first aider could do, which could be put to the panel for rating. These items covered the following broad areas: identification of suicide risk, assessing seriousness of suicide risk, initial assistance, talking with a suicidal person, no-suicide contracts, ensuring safety, confidentiality, and passing time during the crisis. The items are shown in Additional File 1. For the Asian guidelines, we added a few other items based on previous work on suicide prevention in Asian countries $[17,23]$. The initial questionnaire contained 140 first aid action items, plus 13 questions on participants' sociodemographics, experience/training, and opinions on suicide first aid. Open-ended questions to generate additional culturally-specific items were also included. Given that this was an exploratory project, we used Englishlanguage questionnaires, because the cost of doing it in the experts' native languages would have been prohibitive.

\section{Forming the expert panel}

Mental health professionals, including psychiatrists, psychologists, social workers, counselors, and psychiatric nurses currently working in the Philippines, were recruited by DN, HM and EC (see Figure 1) to form the panel. The recruits were a mix of active practitioners from government, private, and non-government organizations providing psychiatric and other psychosocial services, although there was initial preference for active practitioners who were also connected with academia. In order to increase the cultural appropriateness of the guidelines, when forming the expert panels in each country we were careful to include as wide a representation (cultural and geographical) of professionals as possible.

When invitation letters, together with the Plain Language Statement, were sent (by email) to professionals asking them to be involved, they were also invited to nominate any colleagues who they felt would be appropriate panel members. During the recruitment process, potential participants were informed that one of the selection criteria was fluency in written English.
No attempt was made to make panels representative. The Delphi method does not require representative sampling; it requires panel members who are information and experience-rich [15].

The number of panel members in previous Delphi studies has varied considerably from 15 to 60 [24]. We aimed to have a minimum of 25 members in the panel.

\section{Delphi process}

In Round 1 of the Delphi process, panel members were asked to complete an on-line questionnaire. This was administered using SurveyMonkey [25], with the option to complete it by email or paper mail if this was not possible (although no participant opted for this alternative). The questionnaire consisted of a list of first aid actions to rate. Only actions that are do-able by mental health first aiders were included in the list of items to be rated. Members of the expert panel were given the following instructions to guide their judgments:

"The following questionnaire asks about the best way a member of the public can help someone who is thinking about, or planning to suicide. Mental health first aid is defined as help given to someone who is experiencing a mental health problem, or is in a mental health-related crisis, until professional help is received or the crisis resolves. It does not include counseling or therapy. In the case of suicide, mental health first aid is given until the person decides to accept professional help, or decides against suicide. People who offer mental health first aid may be friends, family members, colleagues or acquaintances. They may or may not be involved in the person's life before or after offering first aid. For brevity, we will refer to the person offering assistance as "the first aider". When completing this questionnaire, you will read statements describing possible actions that the first aider can take to assist a suicidal individual. You will be asked to rate how important each item is as a guideline for a first aider. Please rate as "essential" or "important" those items which you feel should guide most people, most of the time, when assisting a suicidal person. The statements in this questionnaire were derived from a search of both professional and lay literature in English-speaking western countries. Therefore, there will be actions which would be appropriate for members of the public in your country which are not included and there will be actions that may be appropriate in Western countries but not in your cultural context. At the bottom of each page, there is room for you to add suggestions. Please consider the cultural, social and religious environment where you live, and try to add some relevant suggestions 


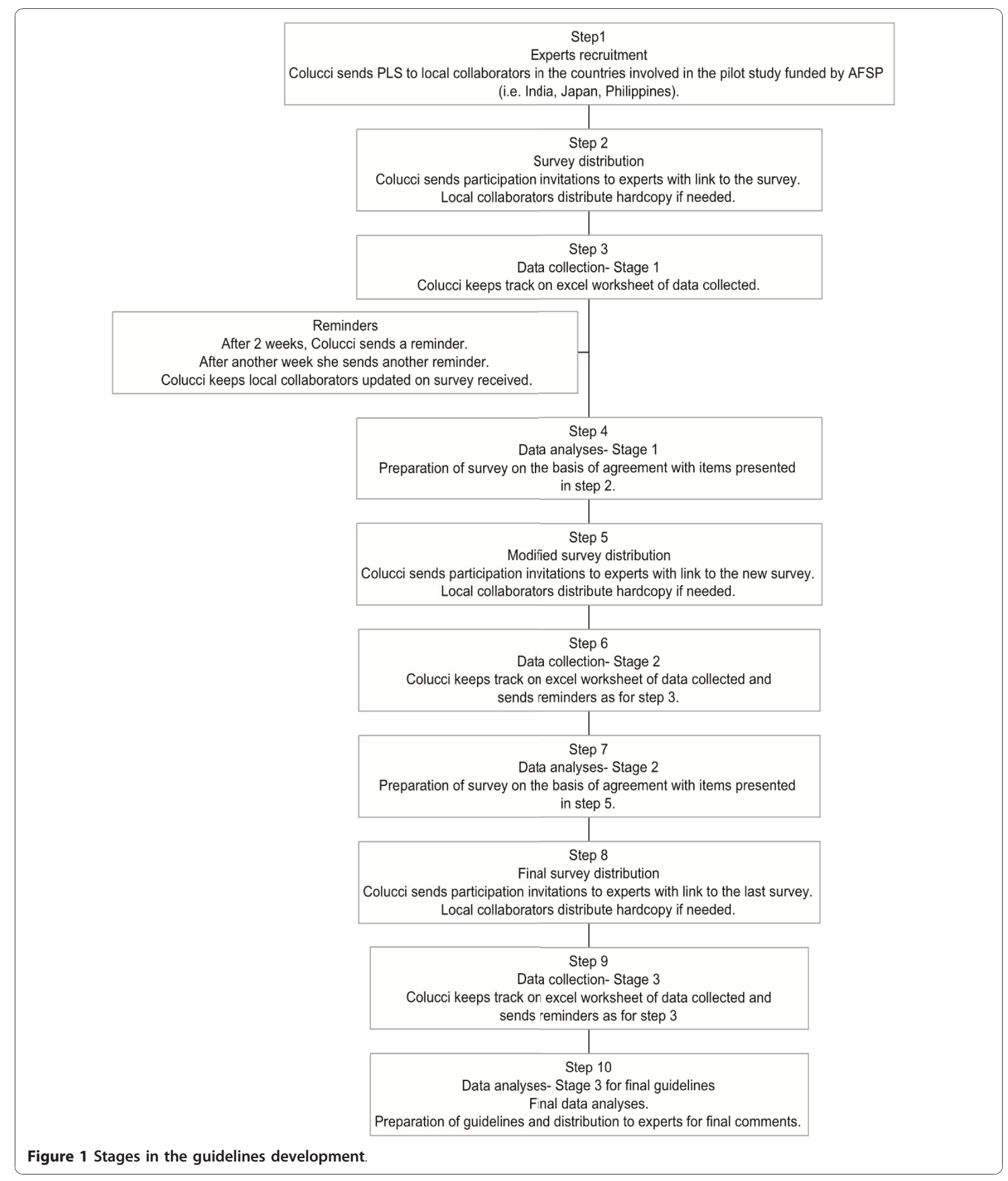

on each page. The more panel members add to this questionnaire, the more relevant and useful the guidelines will be for each individual country. Thank you for taking the time to assist us in this important suicide prevention project!".
The definition of mental health first aid given to the panel in the above instructions distinguishes a first aider's role from that of a clinician. In the case of a suicidal person, the first aider responds by getting professional help for the person, and supporting the person 
and ensuring their safety until the crisis has passed or until the suicidal person is receiving professional treatment and care. The guidelines needed to focus on the immediate prevention of suicide and not on solving the problems that led to the crisis.

Panel members were asked to rate each statement according to how important they believed it was as a potential first aid action for helping a suicidal person. The response scale was: 1 . Essential; 2. Important; 3. Don't know/Depends; 4. Unimportant; 5 . Should not be included. The scale was purposefully asymmetric because only items with positive ratings were of interest for the guidelines. This scale has worked well in previous guideline development work [8].

At the end of each block of items the participants were asked to give any comments. In particular, they were invited to comment on items that were in the initial questionnaire that they considered to be culturally irrelevant or unacceptable, or that would not be feasible because of the local health system and other resources. Panel members were also invited to add any additional actions that were not included in the questionnaire. This was the place where culturally specific material could be introduced. The suggestions made by the panel members in response to the open-ended questions were reviewed by the research team and used to construct new items. Suggestions were accepted and added to Round 2 if they represented a new idea, could be interpreted unambiguously and were actions. Suggestions were rejected if they were near-duplicates of items in the questionnaire, if they were too specific, too general or were more appropriate to therapy than first aid.

Responses were analysed to give the percentage of the panel who rated an item as either "essential" or "important". Items for which there was at least $80 \%$ consensus were included in the guidelines. Items were re-rated if $70-79 \%$ of the panel rated them as "Essential" or "Important".

In Round 2, a second questionnaire was prepared. This consisted of items that were positively rated by at least $70 \%$ of respondents but did not reach the $80 \%$ criterion for inclusion and new items that were generated from the comments in Round 1. A small number of items that received more than $50 \%$ of "Don't know/Depends" or "Not sure" answers were reworded, clarified or specified, and re-rated. For example, "contact the person's spiritual or religious leader" became "contact the person's spiritual or religious leader, if they have one". Participants received an email with an individualized link to the online survey and a Word file that, together with the latter items, fed back a statistical summary of the items that were to be re-rated (i.e. their own original response to the item together with total percentages of endorsement of the item). They were told that they were able to change their responses when re-rating an item if they wished to do so. At the end of this round, any item that reached the $80 \%$ consensus criterion was selected for inclusion in the guidelines, those reaching between 70 $79 \%$ of consensus were re-rated and the rest were rejected. This process was repeated for Round 3.

\section{Ethics}

Ethics approval for the study was obtained from the University of Melbourne Human Research Ethics Committee (Project No. HREC 0605537).

\section{Results \\ Sample}

In the Philippines, 34 panel members were involved in Round 1 (i.e. $54 \%$ of the experts who were invited to participate), 29 in Round 2 and 28 in Round 3. All panel members were currently working in the Philippines. The majority were psychiatrists $(\mathrm{N}=15)$ and psychologists $(N=9)$. Six participants were counselors or counselor advocates, two were social workers and one was a psychiatric nurse. Two of the participants indicated that they were also priests. The panel was comprised of 13 males and 21 females. The majority of the participants were in the age range $40-49$ years $(\mathrm{N}=12)$ and 50-59 years $(\mathrm{N}=12)$, two participants were aged 18-29 years, five were aged 30-39 years, and three were aged 60 years or over.

Some information was also collected on the clinical experience of the panel members. On average, participants reported that they had practiced in mental health/psychiatry for 15 years (the shortest time was 1 year and the longest 31 years). Less than half of the participants $(\mathrm{N}=14)$ received some formal education related to their profession overseas (mainly in USA, Canada and other Asian countries). Slightly over a third of the participants $(\mathrm{N}=12)$ reported having received a formal training specifically on suicide prevention/intervention. However, when asked to state how well prepared they felt to assist a suicidal person, 16 answered "somewhat prepared", 12 "mostly prepared" and 6 "very prepared". No one reported being "not all prepared". Although participants generally felt prepared to assist a suicidal person, in their opinion most people in the Philippines are not at all prepared $(\mathrm{N}=17)$ or somewhat prepared $(\mathrm{N}=17)$. No participant believed others are mostly or very prepared to assist.

\section{Item endorsement}

After three Delphi rounds, there were 102 items that were rated as "essential" or "important" by $80 \%$ or more of the panel members.

At Round 2, 48 new items suggested by participants were added to the questionnaire. The following are examples of such items: 
- An important warning sign for suicide is if a person expresses in words or actions the desire or hope that they will die (including praying that God may take their life).

- An important warning sign for suicide is if a person is not doing usual important tasks, such as household chores, going to work or school.

- The first aider should be aware that the absence of a belief in a God may increase the risk of suicide.

- The first aider should ask the suicidal person if they have experienced a change in their spiritual/religious practices or beliefs (e.g. an increase or decrease in prayer or church attendance).

- The first aider should take the suicidal person in the nearest safe place (e.g. church, hospital, or police station).

- The first aider should ask the suicidal person if they would like the first aider to contact someone for them, such as a friend, family member or trusted religious leader.

- The first aider should consider the suicidal person's spiritual/religious beliefs and refer to these to try to prevent the person from taking their life.

- During the suicidal crisis, the first aider should encourage the suicidal person to spend time with their significant others (e.g. family, friends or religious leader).

A number of responses to the Round 1 open-ended questions did not meet criteria for creation of a new item (e.g. they did not fit the definition of first aid or did not suggest a clear action) or were comments/suggestions. The following are examples of the comments and suggestions that did not generate new items:

- The area where I work is highly multicultural (Christians, Muslims, Indigenous people, other ethnic groups). Mental Health First Aid should consider this aspect as truly essential.

- Suicide is something that needs to be taken seriously. I think everybody should be trained on how to deal with this case as we don't know when it can happen.

- Religious organizations in the Philippines should be oriented and trained on the Mental Health First Aid for Suicide since they serve as powerful influence on the mindset and lifestyle of their followers. Each also has its own idea/perception on suicide.

- There are so many myths about suicide in the Philippines, perpetuated to a great extent by the church and an uneducated field of professionals. A focused training/orientation program for the churches and the helping professions might be a good starting point.

- Local government officials at the community level and the local law enforcement agency are usually called immediately during emergencies, I think they will benefit most with this kind of training.
- (...) will we be given a copy of the guidelines once all data have been gathered and finalized? Will you be conducting a formal training course on the prevention of suicide in Australia? That, I think, would be a very big help to teach experts on the mental health field to put to exercise this paper and put it to a "field test" later on. I am interested to participate and train for such course if this will be considered in the future. Thank you for letting me participate in this study [name deleted].

See the Additional File 1 for a complete list of rated statements, including the percentage of panel members endorsing each item.

At the end of the survey, participants were asked their opinions about the likely effectiveness of suicide first aid, using a 5-point Likert scale (from "definitely yes" to "definitely no"). All the participants who answered the question $(\mathrm{N}=32)$ believed that if the first aider does the right thing the risk of suicide can be reduced. An overwhelming majority of the respondents $(\mathrm{N}=29)$ thought that if the first aider does the wrong thing the risk of suicide can be increased.

The longer-term goal of the project is to use the guidelines to develop, implement and evaluate a training program on suicide first aid in the Philippines. This goal reflects the opinion of the expert panel. When asked if they thought members of the public should receive such training, 25 participants responded "definitely yes" and 4 "probably yes". Only two respondents answered "don't know/depends" and one "probably no".

\section{Development of a guidelines document}

The output from the Delphi process was a set of agreed upon action statements. The statements refer to actions that can be done by a mental health first aider. To be usefully communicated, this list of action statements was transformed into narrative text - the Suicide First Aid Guidelines (see Additional File 2).

The draft guidelines were sent to all panel members for their comments and final endorsement. Since the guidelines were meant to be useful to members of the public, it was important to ensure that they were written to be comprehensible to the target non-professional readership. Feedback from panel members was explicitly sought on the structure and readability of the guidelines, and suggested improvements were incorporated in the final version.

\section{Discussion}

This project has demonstrated that it is possible to achieve consensus among mental health professionals on first aid strategies for suicidal thoughts and behaviour, and that the Delphi method is suitable for developing consensus guidelines in the Philippines. The 
method has been similarly successful in Japan and India. We would suggest that guideline development studies, using a similar method, could be carried in a number of other countries. As well as developing country-specific guidelines, it will also be possible to develop guidelines that are appropriate for cultural minorities within a country. This approach has been used in Australia, with a separate Delphi study undertaken to develop guidelines for Aboriginal Australians, using Aboriginal mental health experts as panel members [26], and specific teaching programs have been developed for non-English speaking immigrant communities [27].

The next steps will be dissemination and use of the guidelines for the purpose of increasing community members' ability to recognize the risk of suicide and undertake basic first aid actions. This will involve translation into local languages and examination of whether modifications are required for cultural minority groups. The guidelines are currently being translated in Filipino (Tagalog) using a back-translation process.

The guidelines that have been developed will serve as a basis for detailed work on culturally appropriate guideline development in languages other than English and for specific national and sub-national cultural groups.

These guidelines can be used as a source of advice to the public, as a basis for determining the curriculum of first aid training courses, and as a standard against which to evaluate the quality of existing materials and programs. The guidelines will inform the development of culturally appropriate training programs and information materials for how a member of the public can assist someone who is suicidal. In some countries and areas with less developed health systems, we believe the guidelines will be useful for primary health workers as well as for members of the public. It is anticipated that the results of this project will contribute to a program of training for community nurses and midwives and for village mental health workers to enable them to contribute more effectively to suicide prevention programs. It will, of course, be necessary to evaluate the impact of such training programs [27-29]. These guidelines could also be useful in the school context. As mentioned earlier, a great part of suicide-related deaths in the country are among young people. Furthermore, the majority of patients admitted to hospital for a suicide attempt are high school students [30]. Considering the higher risk among young people, in 2005 the Student Suicide Prevention Act was enacted, which requires school principals to report all incidents of suicidal behaviour by students in their schools, regardless of whether suicide was completed or not. The law also requires all schools to provide suicide prevention programs for their students. However, a recent investigation [3] in the country's top universities highlighted the absence of suicide prevention programs in the majority of these universities. Where support was offered to students who had been identified as being at suicide risk, it generally consisted of individual counselling. Although several guidance and counselling courses/ workshops are organized in colleges, there does not appear to be any suicide-specific training. These guidelines, which were written also with the help of child psychiatrists and school counsellors, might contribute to the development of such training.

It is important to note that a considerable portion of Filipino participants, in comparison to participants in India and Japan, suggested new items and wrote comments concerning religious and spiritual concepts. This could suggest that, in this country even more than in others, spirituality and religion are powerful forces on suicide and its prevention and must be taken into consideration when developing suicide prevention programs $[23,31]$.

\section{Limitations}

One limitation of this study is the relatively small number of panel members, although Delphi studies have been successfully run even with smaller groups and a size of 20-30 experts is common for this sort of study. Slightly more than half of the professionals approached agreed to participate. However, it must be noted that potential participants were approached via the email address that was available, but several emails bounced, and it is likely that a portion of such addresses were invalid or no longer in use.

While these guidelines have been developed for the Philippines, it is recognised that this country is characterised by considerable cultural and linguistic diversity. It is possible that the guidelines are not applicable to minority cultures within the Philippines.

The questionnaire was administered in English rather than in the panelists' native languages. This of course limits the general applicability of the findings. It is possible that there would have been more culturally specific responses if panelists had used their native language. It is also possible that selecting participants who were fluent in English might have caused the exclusion of experts who were less exposed to the international literature and therefore held more traditional views on suicide. This might explain, for instance, why the majority of the spiritual/religious items that were suggested by some of the experts did not reach the consensus criteria.

Future studies should recruit broader and more representative expert panels including, where possible, professionals from all the relevant mental health disciplines and consumer and carer representatives. This is presently difficult in many Asian countries because there are few clinical psychologists, psychiatric social workers, 
occupational therapists and mental health nurses, and the participation of consumers and carers in such research, as members of an 'expert panel' rather than as research subjects, is still uncommon [11].

Another limitation is that the inclusion of culturally relevant material was dependent on panelists responding to the open-ended questions and not every participant did this. In some cases, this may have been due to lack of time or because the participants in this study were better able to read English than to write it. It may also be because, in questionnaires of all kinds that require ratings to be made, respondents rarely take the opportunity to write comments or to make suggestions when the opportunity is given [11]. However, it must be noted that, compared to other similar mental health first aid guideline research, a considerable number of suggestions for new items were given in this study. This might have been because of the emphasis in the instructions to participants on providing suggestions based on participants' cultural, social and religious settings in each section of the questionnaire.

\section{Conclusions}

There is a growing awareness of suicide as a major public health problem, even though there is a taboo in many societies against discussing it openly [1]. Developing suicide first aid guidelines for community members, and training programs based on these, might also contribute towards changing society's attitudes towards suicide and people who consider suicide.

This study has demonstrated that it is possible to reach consensus for the development of guidelines for the Philippines. Although the guidelines were designed for the public, they may also contain advice that might be helpful to people working in health and welfare professions.

Where the guidelines are used as the basis for first aid training, efforts need to be made to evaluate their impact on the first aider's helping behaviour and on the recipients of the first aid. This will assist researchers to develop an evidence base for mental health first aid and suicide prevention initiatives.

In a WHO news release [2] it was stated that "It's important to realise that suicide is preventable". By collaborating with local experts to agree on a minimum set of suicide first aid actions, and by making such guidelines freely and easily accessible to everyone, we hope to convey the message that suicide is preventable, suicide is everyone's business, and everyone can contribute to its reduction. Members of the general public have a crucial role to play in suicide prevention. Creating opportunities for the public to learn basic first aid actions, and how to implement them when needed, is an important step towards more effective suicide prevention.

\section{Additional material}

Additional file 1: Table of data showing the items included in the Delphi survey and the endorsement levels from the Filipino panel members.

Additional file 2: First aid guidelines for the Philippines. This file may be distributed freely, with the authorship and copyright details intact. Please do not alter the text or remove the authorship and copyright details.

\section{Acknowledgements}

The authors gratefully acknowledge the time, effort and contribution of the following panel members: Rufino L. Ramos, Fr. Francisco R. Bustamante, Fr. Bernardo R. Collera, Lou Evangelista, Julius Pre, Romeo Enriquez, Rommel Montes, Isabel Melgar, Julian R. Montano, Susana A. Estanislao, Hope Abella, Dinah Nadera, Jectofer Cabantin, Darly Coeen Pimental, Wilma Lourdes Blas, Jose Andres Sotto, Bia Uy, Luwalhati Pablo, Rhea Concepcion, Denfred Balleza, June P. Lopez, Willy Reyes, Benita Sta Ana-Ponio, Susan Sabado, Clarette Dy, Remedios Guerrero, Maripaz de Guzman, Eleanor Ronquillo, Cynthia Leynes, Benadette Arcena, Edison Galindez, Ruth Bordado, Carlo Castro, Maria Imelda Batar.

Thanks also to Patrick Ronnelle Maglaya Lorenzo to assist with background information on suicide and suicide prevention in the Philippines.

Funding for the project was provided by the American Foundation for Suicide Prevention, the Centre for International Mental Health (The University of Melbourne). Jorm and Kelly were supported by grants from the National Health and Medical Research Council of Australia and Australian Rotary Health.

Sections of this paper have previously been presented in Colucci E, Kelly CM, Minas H, Jorm AF, Chatterjee S: Mental Health First Aid guidelines for helping a suicidal person: a Delphi consensus study in India. International Journal of Mental Health Systems 2010, 4 http://www.ijmhs.com/content/4/1/4

\section{Author details}

${ }^{1}$ Centre for International Mental Health, Melbourne School of Population Health, The University of Melbourne, Parkville, Victoria 3010, Australia. ${ }^{2}$ Mental Health First Aid Training and Research Program, Orygen Youth Health Research Centre, Centre for Youth Mental Health, The University of Melbourne, Parkville, Victoria 3052, Australia. ${ }^{3}$ Faculty of Management and Development Studies, UP Open University, Los Banos, Laguna, Philippines.

\section{Authors' contributions}

EC recruited clinical experts, revised the existing questionnaire, prepared and administered the on-line surveys for Round 1-3, analysed the data, prepared the draft and final guidelines and wrote the first draft of the manuscript. CMK wrote the former questionnaire and contributed to the revised version, supervised every stage of the project and co-wrote the guidelines. $\mathrm{HM} \mathrm{co-}$ wrote the grant application, recruited the main local collaborators and clinical experts, and reviewed the Round 1 questionnaire and the guidelines. AFJ co-wrote the grant application and developed the method. DN was the main local collaborator in the study and recruited the majority of the clinical experts. All authors contributed to the writing of the manuscript and approved the final version.

\section{Competing interests}

The authors declare that they have no competing interests.

Received: 22 September 2010 Accepted: 20 December 2010 Published: 20 December 2010

\section{References}

1. WHO: World Suicide Prevention Day. 2007 [http://www.who.int/ mediacentre/news/statements/2007/s16/en], [accessed on the 24/04/09]

2. WHO: Suicide huge but preventable public health problem. 2004 [http:// www.who.int/mediacentre/news/releases/2004/pr61/en], [accessed on the 29/04/09].

3. Lorenzo PRM: Protective beliefs against suicide among Filipinos. Master Thesis Ateneo de Manila University, Department of Psychology; 2010. 
4. Tierney RJ: Suicide intervention training evaluation: a preliminary report. Crisis 1994, 15:69-76.

5. Kitchener BA, Jorm AF: Mental health first aid training for the public: evaluation of effects on knowledge, attitudes and helping behavior. BMC Psychiatry 2002, 2:10.

6. Linstone HA, Turoff M: The Delphi method: techniques and applications 2002 [http://is.njit.edu/pubs/delphibook].

7. Snyder-Halpern R, Thompson CB, Schaffer J: Comparison of mailed vs. Internet applications of the Delphi technique in clinical informatics research. Proc AMIA Symp 2000, 809-813.

8. Kelly CM, Jorm AF, Kitchener BA, Langlands RL: Development of mental health first aid guidelines for suicidal ideation and behaviour: a Delphi study. BMC Psychiatry 2008, 8:17.

9. Kelly CM, Jorm AF, Kitchener BA, Langlands RL: Development of mental health first aid guidelines for deliberate non-suicidal self-injury: a Delphi study. BMC Psychiatry 2008, 8:62.

10. Kelly CM, Jorm AF, Kitchener BA: Development of mental health first aid guidelines for panic attacks: a Delphi study. BMC Psychiatry 2009, 9:49.

11. Jorm AF, Minas H, Langlands RL, Kelly ML: First aid guidelines for psychosis in Asian countries: A Delphi consensus study. Int J Ment Health Syst 2008, 2:2.

12. Langlands RL, Jorm AF, Kelly CM, Kitchener BA: First aid for depression: a Delphi consensus study with consumers, carers and clinicians. J Affect Disord 2008, 105:157-165.

13. Hart LM, Jorm AF, Paxton SJ, Kelly CM, Kitchener BA: First Aid for Eating Disorders. Eating Disorders 2009, 17:357-384.

14. Kingston AH, Jorm AF, Kitchener BA, Hides L, Kelly CM, Morgan AJ, Hart LM Lubman DI: Helping someone with problem drinking: Mental health first aid guidelines - a Delphi expert consensus study. BMC Psychiatry 2009, 9.

15. Kelly CM, Jorm AF, Kitchener BA: Development of mental health first aid guidelines on how a member of the public can support a person affected by a traumatic event: a Delphi study. BMC Psychiatry 2010, 10:49.

16. Colucci E: The cultural facet of suicidal behaviour: Its importance and negligence. AeJAMH 2006, 5.

17. Colucci E: Cultural issues in suicide risk assessment. In Suicidal Behavior: Assessment of People-at-risk. Edited by: Kumar U, Mandal MK. New Delhi: SAGE; 2009:

18. Colucci E, Martin G: Ethnocultural aspects of suicide in young people: a systematic literature review. Part 1: Rates and methods of youth suicide. Suicide Life Threat Behav 2007, 37:197-221.

19. Colucci E, Martin G: Ethnocultural aspects of suicide in young people: a systematic literature review. Part 2: Risk factors, precipitating agents, and attitudes toward suicide. Suicide Life Threat Behav 2007, 37:222-237.

20. Colucci E, Kelly CM, Minas H, Jorm AF, Chatterjee S: Mental Health First Aid guidelines for helping a suicidal person: a Delphi consensus study in India. Int I Ment Health Syst 2010, 4.

21. Hawgood J, DeLeo D: Suicide Prevention Skills Training Manual: An Accredited Training Program. Brisbane, Australia: Griffith University; 2002.

22. Mental Health and Drug and Alcohol Office: Mental Health for Emergency Departments - A Reference Guide. Sydney: NSW Department of Health; 2002.

23. Colucci E: Recognizing spirituality in the assessment and prevention of suicidal behaviour. WCPRR 2008, 3:77-95.

24. Hasson F, Keeney S, McKenna H: Research quidelines for the Delphi survey technique. J Adv Nurs 2000, 32:1008-1015.

25. SurveyMonkey. [http://www.surveymonkey.com]

26. Hart $L M$, Jorm AF, Kanowski $L G$, Kelly CM, Langlands RL: Mental health first aid for Indigenous Australians: using Delphi consensus studies to develop guidelines for culturally appropriate responses to mental health problems. BMC Psychiatry 2009, 9:47.

27. Minas H, Colucci E, Jorm AF: Evaluation of Mental Health First Aid training with members of the Vietnamese community in Melbourne. Int J Ment Health Syst 2009, 3:19.

28. Jorm AF, Kitchener BA, Mugford SK: Experiences in applying skills learned in a Mental Health First Aid training course: a qualitative study of participants' stories. BMC Psychiatry 2005, 5:43.

29. Kitchener BA, Jorm AF: Mental health first aid training: review of evaluation studies. Aust N Z J Psychiatry 2006, 40:6-8.

30. Gallardo-Lagasca M, Gonong E, Tamesis A: Suicide attempts in adolescents: a call for understanding and action. Philippine Children's Medical Center Journal 2001, 3:39-48.
31. Ruben Tanseco SJ: Psychiatry and Spirituality. Paper delivered at the Philippine Psychiatric Association Midyear Convention. Philippines 2007.

doi:10.1186/1752-4458-4-32

Cite this article as: Colucci et al: Mental Health First Aid guidelines for helping a suicidal person: a Delphi consensus study in the Philippines. International Journal of Mental Health Systems 2010 4:32.

\section{Submit your next manuscript to BioMed Central and take full advantage of:}

- Convenient online submission

- Thorough peer review

- No space constraints or color figure charges

- Immediate publication on acceptance

- Inclusion in PubMed, CAS, Scopus and Google Scholar

- Research which is freely available for redistribution

Submit your manuscript at www.biomedcentral.com/submit
Biomed Central 